\title{
Sarcopenia Measured by Temporalis Muscle Thickness Independently Predicts Early Relapse and Short Survival in Primary CNS Lymphoma
}

\section{Alipi Bonm}

University of Washington https://orcid.org/0000-0003-0340-2008

Anthony Menghini

University of Washington

Jerome J. Graber ( $\nabla$ jgraber@uw.edu )

University of Washington

\section{Research Article}

Keywords: Sarcopenia, temporalis muscle, TMT, PCNSL

Posted Date: November 30th, 2021

DOI: https://doi.org/10.21203/rs.3.rs-1082843/v1

License: (c) (i) This work is licensed under a Creative Commons Attribution 4.0 International License.

Read Full License 


\section{Abstract}

Introduction: Primary CNS lymphoma (PCNSL) outcomes diverge between a majority of patients who achieve long term remission and a smaller minority who have aggressive disease course and die in the first year. Sarcopenia is increasingly recognized as a powerful predictor of mortality in brain and systemic cancers. Temporalis muscle thickness (TMT) is a validated radiographic measure of sarcopenia. We hypothesized that patients with TMT less than one standard deviation below the mean ("very thin TMT") would go on to have shorter survival.

Methods: Two blinded operators retrospectively measured TMT in 99 consecutive pretreatment brain MRIs from patients that were subsequently diagnosed with PCNSL.

Results: On univariate analysis TMT predicted early progression (HR 4.25, 95\% Cl $1.95-9.29, \mathrm{p}<0.001)$ and early mortality (HR 4.38, 95\% Cl $2.25-8.53, p<0.001$ ), and these effects were maintained in subgroups of patients both $<65$ and ${ }^{3} 65$ years of age. Very thin TMT predicted mortality more robustly than IELSG or MSKCC scores. Patients with very thin TMT received fewer cycles of high-dose methotrexate (HD-MTX) and were less likely to receive consolidation. On multivariate analysis which included the covariates age, sex, TMT, ECOG, BMI, lifetime doses of HD-MTX, and consolidation, very thin TMT was independently associated with both early progression $(\mathrm{HR}=7.87,95 \% \mathrm{Cl}=3.55-17.45$, $\mathrm{p}<0.001)$ and short survival (HR 4.49, 95\% $\mathrm{Cl}=1.94-10.40, \mathrm{p}<0.001)$.

Conclusions: We conclude that PCNSL patients with very thin TMT are at high risk for relapse and early mortality. Future trials should stratify patients by TMT to avoid potential confounding.

\section{Introduction}

Primary CNS lymphoma is unusual among brain cancers in that long-term durable remissions can be achieved in a large proportion of patients. With autologous stem cell transplant (ASCT) consolidation, approximately $60-70 \%$ of patients will remain relapse-free after 5 years [1, 2], although late relapses are known to occur [3]. Therefore intensive, and frequently in-hospital treatment regimens are justified by the potential for long-term remission. On the other hand, approximately $10-20 \%$ of patients will be refractory to initial induction with high-dose methotrexate (HDMTX) based regimens $[4,5]$ with a median survival of 2.1 months after progression [6]. Additionally, patients who relapse within the first year had a one-year survival of about $20 \%$, whereas patients who relapsed more than a year after diagnosis had a one-year post-relapse survival of greater than $70 \%$ [6]. Unfortunately, relapse is most common in the first year, occurring in $85 \%$ of patients that are not consolidated, and in $59 \%$ of patients who are consolidated after first induction. [6]. Importantly, even patients with refractory disease or early relapse can achieve longterm progression-free survival if a second remission can be achieved [7], and we previously reported that there is no correlation between the durability of successive responses [4]. Overall, the one-year mortality of PCNSL is approximately $21-24 \%[8,9]$. Thus, it is important to be able to reliably and easily stratify 
patients at the time of diagnosis, in order to individually tailor treatment strategies as well as to ensure balancing of prognostic factors in clinical trials.

Sarcopenia describes reduced muscle mass and function and is increasingly recognized as an important prognostic feature in oncology [10]. The etiology of sarcopenia is thought to be a combination of agerelated muscle loss as well as cancer related effects of chronic inflammation and increased protein degradation. As such, it is unsurprising that sarcopenia has been linked with poor prognosis [11] as well as reduced ability to tolerate chemotherapy [12]. For example, in systemic diffuse large B cell lymphomas, sarcopenic patients have been shown to have two year survival of $46 \%$, compared with $84 \%$ in nonsarcopenic patients [13].Temporalis muscle thickness (TMT) has become accepted as a radiographic measure of sarcopenia which is strongly correlated to survival in multiple neurologic diseases, including glioblastoma [14, 15]. TMT is also been validated as highly predictive of grip strength, another frequently used measure of sarcopenia [16]. A study earlier this year reported that reduced TMT is associated with shorter survival in patients with PCNSL [17], using predetermined gender specific standard values from healthy individuals aged 18-40 [16]. We hypothesized that rather than comparing to healthy patients, it would be preferable to use a single cutoff to identify PCNSL patients (most of whom are over 40) with the lowest TMT, and that this population would ultimately go on to have short survival times. One standard deviation was chosen because this would isolate the approximately $16 \%$ of patients with the thinnest TMT, which we hypothesized would significantly overlap with the $20 \%$ of patients that have aggressive disease and die within a year of diagnosis.

\section{Methods}

This study was approved by the University of Washington institutional review board. A database of all PCNSL patients treated at the University of Washington between 2000-2021 was created combining an unbiased screen for ICD9/10 codes as previously reported [4]. We retrospectively reviewed the presenting brain MRIs for all patients diagnosed from 2010 through 2021. A total of 135 patients were screened, and 36 patients were excluded due to lack of pretreatment MRI images, motion artifact, poor quality images, or missing clinical data.

Temporalis muscle thickness was measured by a board-certified neuro-oncologist (AVB) and a medical student (AM) who were blinded to clinical history at the time of measurement. Measurements were performed as follows: in two windows, an axial pre-contrast T1 image and corresponding coronal image were placed side to side, the axial images were chosen at the level of the superior aspect of the anterior commissure (on coronal sections) corresponding to the level of the Sylvian fissure (Figure 1). The thickness of the temporalis muscle belly was measured perpendicularly to the long axis of the muscle. The final measure was the average of left and right temporalis muscle thickness obtained by both observers. If there was any evidence of skull defect or other intervention on one side, the measurements from this side were discarded. 
Demographic, pathologic, laboratory, and outcomes data were retrospectively obtained from the medical records in this no-contact study. Overall survival and progression-free survival were calculated starting from the date of diagnosis, through to either last contact, date of death, or date of progression. Univariate Cox regressions were performed in SPSS (IBM, Armonk, NY) with an alpha of 0.05. In order to check for confounding by covariates which might reasonably be expected to be related to sarcopenia (age, sex, $\mathrm{BMI}$ ) or outcome (performance status, age, number of HD-MTX doses, and consolidation), we performed a multivariate analysis in SPSS with alpha 0.05. Plots were generated in Prism 8.0 (Graphpad, San Diego, $\mathrm{CA})$.

\section{Results}

Patient characteristics are described in Table 1. A total of 99 consecutive patients with PCNSL were identified between 2010 and 2021 for whom pre-treatment brain MRI was available for review. Patients were $41 \%$ female and $58 \%$ male, with median age at diagnosis of 67 years. Average BMI was $29.4 \mathrm{~kg} / \mathrm{m} 2$, and median ECOG was 1. Patients were treated with a median of 6.5 cycles of HD-MTX and were consolidated as follows: 18 patients were consolidated with myeloablative chemotherapy followed by autologous stem cell transplant, 10 patients received WBRT, 4 patients were consolidated with etoposide and cytarabine [18], and 67 patients were not consolidated, which includes patients treated with maintenance chemotherapy, targeted agents (ex. Ibrutinib), or immunomodulatory agents (lenalidomide). A total of 52 patients progressed and 42 patients died. Median duration of follow up was 33.3 months. Patients with reduced TMT received a median of 2 doses of HD-MTX throughout the course of their treatment, whereas other patients received a median of 6.5 doses of HD-MTX. With respect to consolidation strategy, only $1 / 15$ patients with reduced TMT was consolidated (with nonmyeloablative cytarabine and etoposide) whereas the remaining 14 were either not consolidated or received maintenance treatments. In contrast, 18/84 patient with normal TMT were consolidated with autologous stem cell transplant, 9 were consolidated with WBRT, 4 received nonmyeloablative cytarabine and etoposide, and 53 received no consolidation or maintenance therapies.

Interobserver agreement for TMT measurements was interrogated using Bland-Altman analysis which revealed a bias of 0.03 , calculated as the difference between the measurements from each operator, divided by the average measurement. TMT was normally distributed and fitted to a Gaussian distribution. Mean temporalis thickness was $7.8 \mathrm{~mm}$ with standard deviation of $1.8 \mathrm{~mm}$. Patients with TMT less than one standard deviation below the mean (corresponding to $<6.0 \mathrm{~mm}$ ) were grouped as "very thin TMT" for all subsequent analyses. Males had significantly thicker TMT compared to females, average $8.3 \mathrm{~mm}$ and $7.5 \mathrm{~mm}$ respectively (Fisher's T-test, two tailed: $\mathrm{p}=0.044$ ). TMT showed a nonsignificant trend towards inverse correlation with age $\left(r^{2}=0.036, p=0.059\right)$, which was unchanged when including only patients age $\geq 65\left(r^{2}=0.037, p=0.174\right)$.

Currently accepted prognostic scores include the International Extranodal Lymphoma Study Group (IELSG) score [19] and the Memorial Sloan Kettering Cancer Center (MSKCC) prognostic model [9]. IELSG scores could be calculated for $57 / 99$ patients, due to missing datapoints in the other patients. In this 
smaller cohort, we were unable to detect a significant difference in survival (Figure 2A; $p=0.394$ ) between IELSG 0-1, 2-3, and 4-5. [19] We also analyzed patients according to the MSKCC score, which divides patients into three groups based on age and KPS. We detected a significant difference in survival between the three groups (Figure $2 B, p=0.047$ ), specifically there was decreased survival in patients aged $>50$ years and with $\mathrm{KPS}<70(\mathrm{HR}=4.88,95 \% \mathrm{Cl}=1.84-12.91, \mathrm{p}=0.018)$, and a nonsignificant trend towards decreased survival in patients aged $>50$ years and with $\mathrm{KPS} \geq 70(\mathrm{HR}=2.32,95 \% \mathrm{Cl}=0.93-$ $5.77, p=0.070$ ). In comparison, when stratifying patients by TMT, we found that thin TMT was highly predictive of both shorter survival (Figure 1B; HR 4.38, 95\% Cl 2.25-8.53, p < 0.001) and shorter progression-free survival (Figure 1C; HR 4.25, 95\% Cl 1.95-9.29, p < 0.001). Of note, all 15 patients with very thin TMT had progressed by 13.2 months, and 1 year survival was $5 / 15(33 \%)$. We subsequently analyzed patients $\geq 65$ years $(n=52)$ and stratified them by TMT. We again found shorter survival in patients with thin TMT (Figure $2 \mathrm{~A} ; \mathrm{HR}=2.32,95 \% \mathrm{Cl}=1.06-5.10, \mathrm{p}=0.036$ ) and shorter progressionfree survival (Figure 2B; $\mathrm{HR}=3.71,95 \% \mathrm{Cl}=1.72-8.01, \mathrm{p}<0.001$ ). These effects were maintained when analyzing only patients $<65$ years $(n=47)$, for both survival (Figure $2 C ; \mathrm{HR} 11.1,95 \% \mathrm{Cl}=3.2-38.7$, $p<0.001$ ) and progression-free survival (Figure 2D; $\mathrm{HR}=27.5,95 \% \mathrm{Cl}=5.9-128.0, p<0.001$ ).

Next, we explored the association between BMI and outcomes (Supplementary Figure 1). We hypothesized that elevated BMI would predict shorter survival, and this effect was indeed observed (HR $2.05,95 \% \mathrm{Cl}=1.10-3.82, \mathrm{p}=0.028)$. We subsequently hypothesized that the population of patients with high BMI but low TMT would identify a population of patients with baseline obesity and concurrent sarcopenia. We generated an obesity-sarcopenia index calculated as TMT (mm) / BMI *10. The mean and standard deviation were 2.76 and 7.55 , respectively. Patients with an obesity-sarcopenia index of less than $\mu-\sigma$ were designated as $\mathrm{BMI}_{\text {high }} / \mathrm{TMT}_{\text {low. }}$. There was a nonsignificant trend for shorter survival in $\mathrm{BMI}_{\text {high }} / \mathrm{TMT}_{\text {low }}$ patients $(\mathrm{HR} 1.84,95 \% \mathrm{Cl}=0.75-0.453, \mathrm{p}=0.098)$.

In order to control for confounding by covariates, we performed multivariate analysis including TMT, age, sex, ECOG, BMI, lifetime number of HD-MTX doses, and consolidation as covariates (Table 2). The Cox multivariate model was significant for overall survival $\left(X^{2}=52.12, p<0.001\right)$. Of the individual covariates, TMT (HR 4.49, 95\% Cl = 1.94-10.40, $p<0.001)$, lifetime HD-MTX doses $(H R=0.82,95 \% \mathrm{Cl}=0.74-0.91$, $\mathrm{p}<0.01)$, consolidation $(\mathrm{HR}=0.15,95 \% \mathrm{Cl}=0.04-0.66, \mathrm{p}=0.012)$ and $\mathrm{BMI}(\mathrm{HR} 1.05,95 \% \mathrm{Cl}=1.01-1.10$, $\mathrm{p}=0.027$ ) were independently correlated with survival (see Table 2 ). A separate Cox multivariate model was run for progression-free survival $(\chi 2=38.15, p<0.001)$. Only TMT $(H R=7.87,95 \% \mathrm{Cl}=3.55-17.45$, $p<0.001)$ and $\mathrm{BMI}(\mathrm{HR} 1.05,95 \% \mathrm{Cl}=1.01-1.09, \mathrm{p}=0.016)$ were independently associated with progression-free survival (see full results in Table 2).

\section{Discussion}

In this study we demonstrate that patients with sarcopenia as measured by TMT less than $6 \mathrm{~mm}$ (defined by $\mu-\sigma$ in our cohort) on their pretreatment MRIs have much worse prognosis independent of previously described factors. Very thin TMT independently and robustly predicted early progression as well as early mortality in patients across age groups. The independent effect of TMT on PFS suggests that in addition 
to being a marker of fragility, TMT could also be indicative of an aggressive disease state. It would follow that this measure may, in ways not fully understood, integrate both patient-specific and disease-specific characteristics. Although, we also found that patients with very thin TMT had less chemotherapy and were less likely to receive consolidation, these factors were included as covariates in our multivariate models, and therefore the effect of TMT on progression is independent of treatment. The value of thin TMT as a robust predictor is that it can be applied to virtually all patients, since all patients will have a pretreatment MRI in the modern era, and it more robustly predicted survival compared to previously validated prediction scores. If validated in a prospective cohort, this may have utility in patients where the clinician must decide between aggressive management with the ultimate goal of long-term remission, or less aggressive outpatient treatment aimed at palliative control.

Of note, Furtner et al reported an association between TMT and survival in PCNSL earlier this year.[17] Their approach was to generate age and gender specific normal values in a healthy cohort of adults aged 18-40, and then identify those patients with PCNSL who deviated by 2.5 standard deviations from the mean. They found that patients with lower TMT went on to have shorter survival compared to patients with higher TMT, a finding which we now confirm and extend. Our approach was to use TMT to attempt to identify the approximately $20 \%$ of patients who die within a year of diagnosis. To do this we opted to compare TMT within-cohort rather than using normal controls, since the relevant question is to identify the subgroup of poor responders from the cohort of patients with PCNSL. Furthermore, since age and sex have independent effects on survival in PCNSL, we opted to treat TMT independently. This approach succeeded in identifying a small group of patients who had much earlier relapse and shorter survival compared to the overall cohort.

Our study has significant limitations. By their nature, retrospective uncontrolled studies are prone to bias. Additionally, MRI technique was not standardized in this retrospective cohort, and this could potentially have affected TMT measurements. Furthermore, the optimal cutoff value for TMT can be debated, and needs validation in a prospectively collected cohort. Overall, our study can be interpreted as providing category 4 evidence that very thin TMT predicts early relapse and short survival. In addition, clinical trials in PCNSL should incorporate this prognostic factor to avoid imbalance between treatment arms.

\section{Declarations}

\section{Compliance With Ethical Standards:}

Ethical approval: All procedures performed in studies involving human participants were in accordance with the ethical standards of the institutional and/or national research committee and with the 1964 Helsinki declaration and its later amendments.

Funding: The authors report no relevant funding for this study.

Competing Interests: The authors have no relevant financial or non-financial interests to disclose. 
Author contribution: JJG conceived of the study. AVB created the patient database. AVB and AM collected the data. AVB analyzed the data and wrote the manuscript. JJG, AVB and AM edited the final figures and manuscript.

Data availability: The datasets generated during and analyzed during the current study are available from the corresponding author on reasonable request.

\section{References}

1. Kasenda B, Ihorst G, Schroers R, et al (2017) High-dose chemotherapy with autologous haematopoietic stem cell support for relapsed or refractory primary CNS lymphoma: a prospective multicentre trial by the German Cooperative PCNSL study group. Leukemia 31:2623-2629. https://doi.org/10.1038/leu.2017.170

2. Houillier C, Taillandier L, Dureau S, et al (2019) Radiotherapy or Autologous Stem-Cell Transplantation for Primary CNS Lymphoma in Patients 60 Years of Age and Younger: Results of the Intergroup ANOCEF-GOELAMS Randomized Phase II PRECIS Study. J Clin Oncol 37:JC0.18.00306. https://doi.org/10.1200/jco.18.00306

3. Nayak L, Hedvat C, Rosenblum MK, et al (2011) Late relapse in primary central nervous system lymphoma: clonal persistence. Neuro-oncology 13:525-9. https://doi.org/10.1093/neuonc/nor014

4. Bonm AV, Gibson AW, Holmberg LA, et al (2021) A single-center retrospective analysis of outcome measures and consolidation strategies for relapsed and refractory primary CNS lymphoma. J Neurooncol 151:193-200. https://doi.org/10.1007/s11060-020-03648-9

5. Grommes C, DeAngelis LM (2017) Primary CNS Lymphoma. J Clin Oncol 35:JC0.2017.72.760. https://doi.org/10.1200/jco.2017.72.7602

6. Langner-Lemercier S, Houillier C, Soussain C, et al (2016) Primary CNS lymphoma at first relapse/progression: characteristics, management, and outcome of 256 patients from the French LOC network. Neuro-oncology 18:1297-1303. https://doi.org/10.1093/neuonc/now033

7. Soussain C, Hoang-Xuan K, Taillandier L, et al (2008) Intensive Chemotherapy Followed by Hematopoietic Stem-Cell Rescue for Refractory and Recurrent Primary CNS and Intraocular Lymphoma: Société Française de Greffe de Moëlle Osseuse-Thérapie Cellulaire. J Clin Oncol 26:2512-2518. https://doi.org/10.1200/jco.2007.13.5533

8. Bromberg JEC, Issa S, Bakunina K, et al (2019) Rituximab in patients with primary CNS lymphoma (HOVON 105/ALLG NHL 24): a randomised, open-label, phase 3 intergroup study. Lancet Oncol 20:216-228. https://doi.org/10.1016/s1470-2045(18)30747-2

9. Abrey LE, Ben-Porat L, Panageas KS, et al (2006) Primary Central Nervous System Lymphoma: The Memorial Sloan-Kettering Cancer Center Prognostic Model. Journal of Clinical Oncology 24:57115715. https://doi.org/10.1200/jco.2006.08.2941

10. Joglekar S, Nau PN, Mezhir JJ (2015) The impact of sarcopenia on survival and complications in surgical oncology: A review of the current literature. J Surg Oncol 112:503-509. 
https://doi.org/10.1002/jso.24025

11. Martin L, Birdsell L, MacDonald N, et al (2013) Cancer Cachexia in the Age of Obesity: Skeletal Muscle Depletion Is a Powerful Prognostic Factor, Independent of Body Mass Index. J Clin Oncol 31:1539-1547. https://doi.org/10.1200/jco.2012.45.2722

12. Ryan AM, Power DG, Daly L, et al (2016) Cancer-associated malnutrition, cachexia and sarcopenia: the skeleton in the hospital closet 40 years later. P Nutr Soc 75:199-211. https://doi.org/10.1017/s002966511500419x

13. Lanic H, Kraut-Tauzia J, Modzelewski R, et al (2013) Sarcopenia is an independent prognostic factor in elderly patients with diffuse large B-cell lymphoma treated with immunochemotherapy. Leukemia Lymphoma 55:817-823. https://doi.org/10.3109/10428194.2013.816421

14. Hsieh K, Hwang ME, Estevez-Inoa G, et al (2019) Temporalis muscle width as a measure of sarcopenia correlates with overall survival in patients with newly diagnosed glioblastoma. J Radiat Oncol 8:379-387. https://doi.org/10.1007/s13566-019-00408-9

15. Chung SJ, Kim YJ, Yoo HS, et al (2021) Temporalis Muscle Thickness as an Indicator of Sarcopenia Is Associated With Long-term Motor Outcomes in Parkinson's Disease. Journals Gerontology Ser. https://doi.org/10.1093/gerona/glab082

16. Steindl A, Leitner J, Schwarz M, et al (2020) Sarcopenia in Neurological Patients: Standard Values for Temporal Muscle Thickness and Muscle Strength Evaluation. J Clin Medicine 9:1272. https://doi.org/10.3390/jcm9051272

17. Furtner J, Nenning K-H, Roetzer T, et al (2021) Evaluation of the Temporal Muscle Thickness as an Independent Prognostic Biomarker in Patients with Primary Central Nervous System Lymphoma. Cancers 13:566. https://doi.org/10.3390/cancers13030566

18. Rubenstein JL, Hsi ED, Johnson JL, et al (2013) Intensive Chemotherapy and Immunotherapy in Patients With Newly Diagnosed Primary CNS Lymphoma: CALGB 50202 (Alliance 50202). J Clin Oncol 31:3061-3068. https://doi.org/10.1200/jco.2012.46.9957

19. Ferreri A, Blay J-Y, Reni M, et al (2003) Prognostic Scoring System for Primary CNS Lymphomas: The International Extranodal Lymphoma Study Group Experience. Journal of Clinical Oncology 21:266272. https://doi.org/10.1200/jco.2003.09.139

\section{Tables}

Table 1: Patient characteristics. 


\begin{tabular}{ll} 
Total patients & 99 \\
\hline Age at dx, median yrs (range) & $76(19-90)$ \\
\hline Sex (M/F) & $58 / 41$ \\
\hline ECOG (range) & $1(0-3)$ \\
\hline Died & 42 \\
\hline Progressed & 51 \\
\hline Median lifetime cycles HD-MTX (range) & $6.5(0-24)$ \\
\hline Consolidated & $32 / 99$ \\
\hline \multicolumn{1}{c}{ ASCT } & $18 / 32$ \\
\hline \multicolumn{1}{c}{ WBRT } & $10 / 32$ \\
\hline \multicolumn{1}{c}{ Etop/AraC } & $4 / 32$ \\
\hline Not consolidated & $67 / 99$
\end{tabular}

Table 2: Multivariate Cox Regression.

\begin{tabular}{lllllll} 
& PFS & \multicolumn{3}{c}{ OS } \\
\hline Variable tested & HR & $95 \% \mathrm{Cl}$ & P-value & HR & $95 \% \mathrm{Cl}$ & P-value \\
\hline TMT (very thin / other) & 7.87 & $3.55-17.45$ & $<0.001$ & 4.49 & $1.94-10.40$ & $<0.001$ \\
\hline Lifetime HD-MTX doses & 9.39 & $0.88-1.00$ & 0.055 & 0.82 & $0.74-0.91$ & $<0.001$ \\
\hline Consolidation (yes / no) & 0.84 & $0.41-1.75$ & 0.646 & 0.15 & $0.04-0.66$ & $\mathbf{0 . 0 1 2}$ \\
\hline BMI & 1.05 & $1.01-1.09$ & $\mathbf{0 . 0 1 6}$ & 1.05 & $1.01-1.10$ & $\mathbf{0 . 0 2 7}$ \\
\hline Age & 1.02 & $0.99-1.05$ & 0.203 & 1.02 & $0.99-1.06$ & 0.146 \\
\hline Sex (F / M) & 0.57 & $0.30-1.09$ & 0.087 & 0.52 & $0.25-1.09$ & 0.084 \\
\hline ECOG (0-1 / 2-4) & 0.78 & $0.40-1.54$ & 0.483 & 0.758 & $0.35-1.63$ & 0.479
\end{tabular}

Figures 

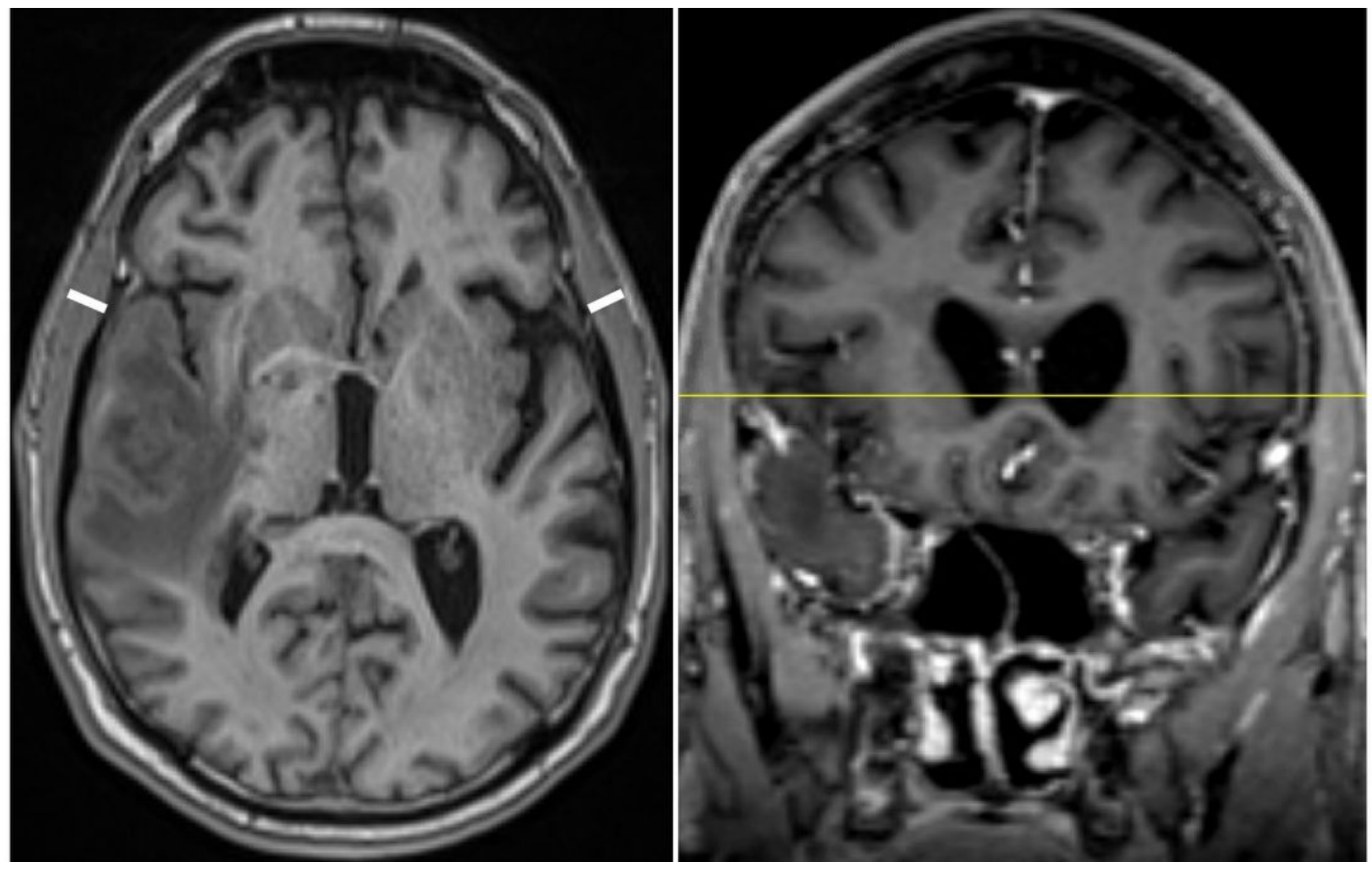

\section{Figure 1}

Example of TMT Measurement. Axial (left) and coronal (right) T1 weighted MRI pre-treatment images are shown for a patient with PCNSL. The axial slice corresponding to the level of the top of the anterior commissure on coronal (yellow line) was used for TMT measurements (thick white lines). 
A
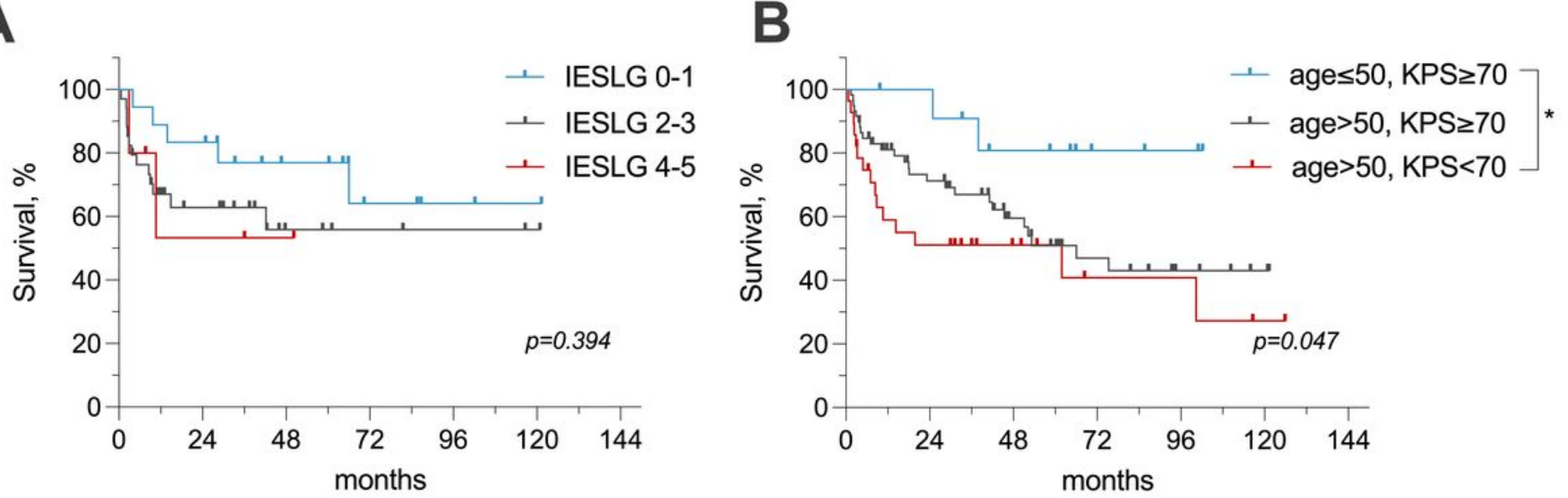

C

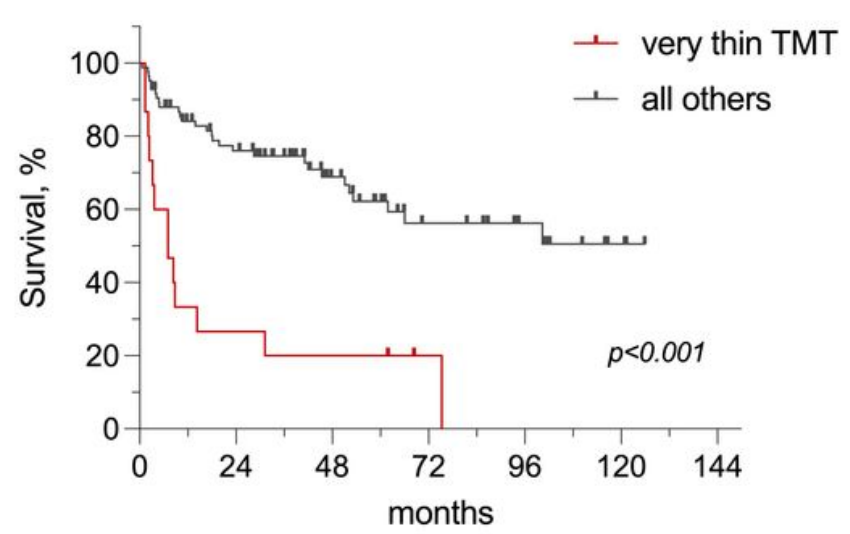

D

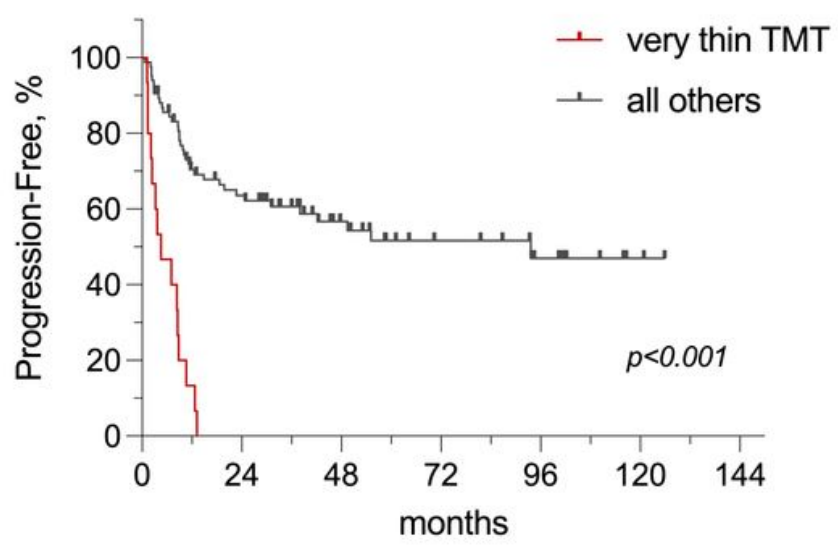

Figure 2

TMT measurement outperforms other prognostic indicators. IELSG (A) and MSKCC (B) prognostic scores were used to stratify patients as previously described. In contrast, very thin TMT more robustly predicted poor survival (C) and early relapse (D). ${ }^{*} \mathrm{p}<0.05$ 
A

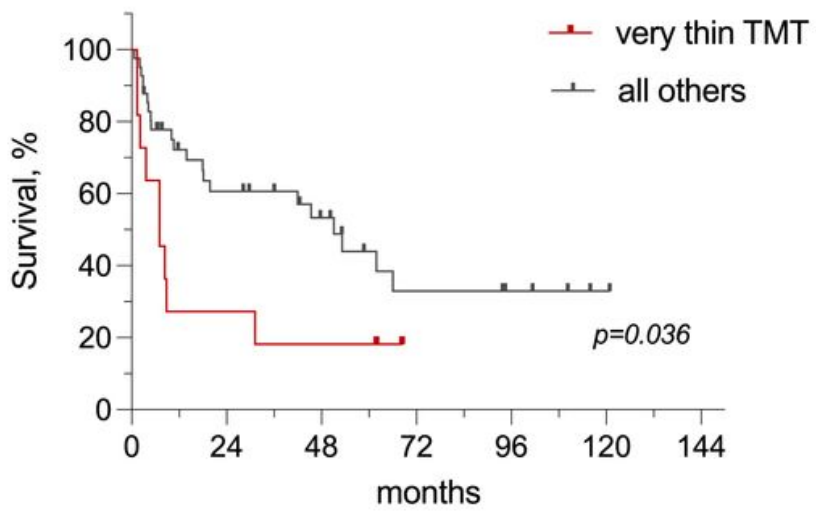

C

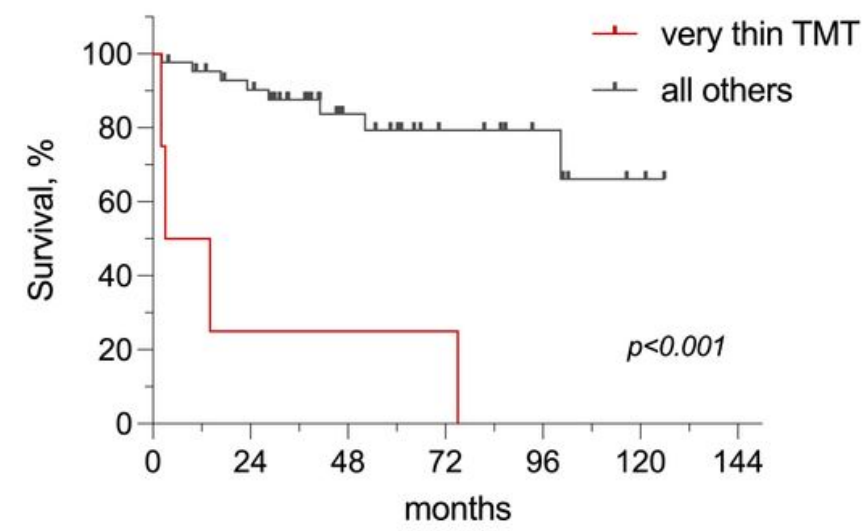

B

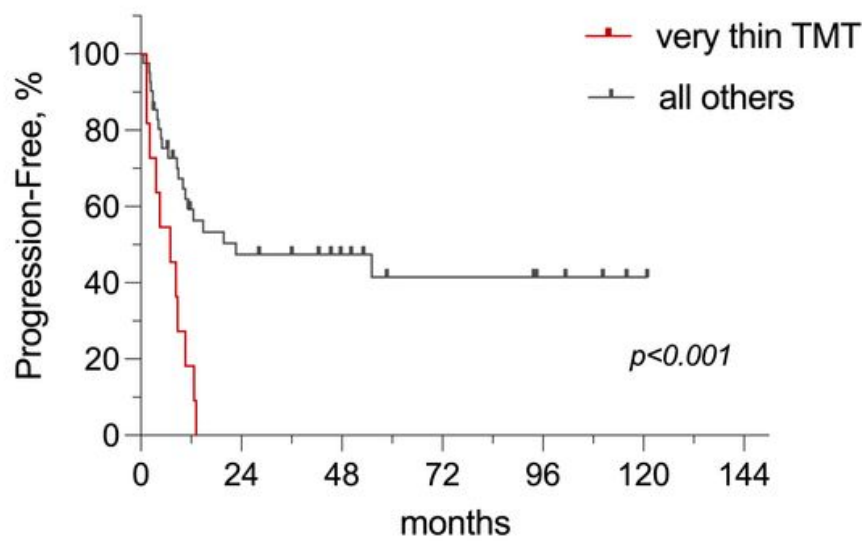

D

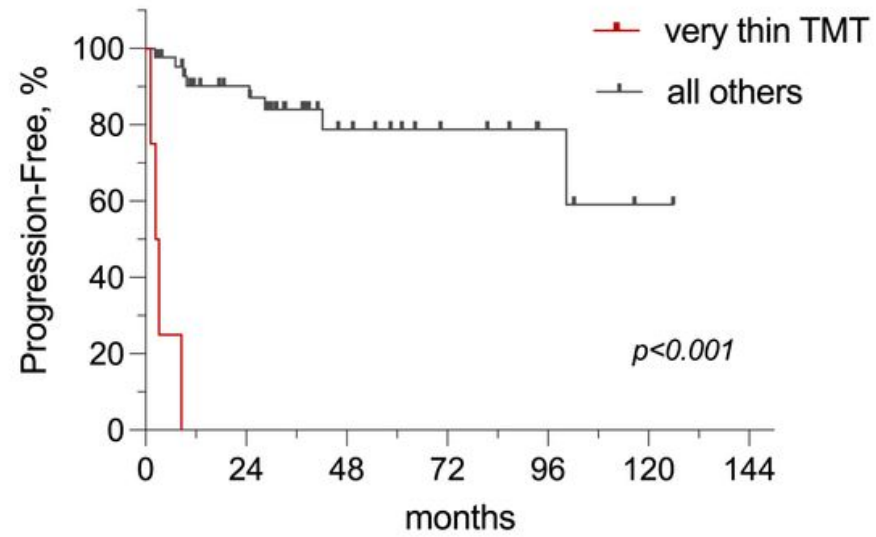

Figure 3

TMT is a robust predictor of outcomes in both elderly and young patients with PCNSL. Patients were separated by age ${ }^{3} 65(A, B)$ or age $<65(C, D)$. TMT predicted poor survival $(A, C)$ and early relapse $(B, D)$ in both age cohorts.

\section{Supplementary Files}

This is a list of supplementary files associated with this preprint. Click to download.

- FigS1BMI.jpg 